\title{
Physical-Layer Network Coding in Coded OFDM Systems with Multiple-Antenna Relay
}

\author{
Meng Wu, Dirk Wübben, Armin Dekorsy \\ Department of Communications Engineering \\ Otto-Hahn-Allee 1, University of Bremen, 28359 Bremen, Germany \\ Email: $\{w u, w u e b b e n$, dekorsy $\} @$ ant.uni-bremen.de
}

\begin{abstract}
In this paper physical layer network coding (PLNC) in two-phase two-way relaying networks using coded orthogonal frequency division multiplexing (OFDM) transmission is investigated. After receiving the superimposed signal from both sources, the relay estimates the XOR-based network coded signal, which is broadcast back to the sources. Assuming that the relay is equipped with multiple antennas, the uplink transmission forms a multiple-input multiple-output (MIMO) system, which allows the application of MIMO detection technologies. To this end, the impact of employing multiple antennas at the relay on different detection and decoding schemes under investigations is studied and compared with respect to mutual information (MI). Numerical simulations verify our theoretical analysis.
\end{abstract}

\section{INTRODUCTION}

Physical layer network coding (PLNC) attracts increasing interest for two-phase two-way relaying networks in achieving improved spectral efficiency [1], [2]. In the multiple-access (MA) phase, both sources transmit simultaneously, resulting in a superimposed received signal at the relay. In order to handle such a MA problem, one possible solution is that the relay estimates the bitwise modulo-2 (XOR) of the two source messages, which is sent back to the sources in the broadcast (BC) phase. The concept of PLNC was jointly considered with channel coding for repeat accumulate (RA) codes in [3] and for low-density parity-check (LDPC) codes in [4] based on aposterior probability (APP) detection. Different APP-based decoding schemes to either first decode the individual messages from the sources explicitly or directly estimate the network coded packet from the receive signal were investigated in [5] based on mutual information.

The previously mentioned literatures employed only singleantenna relay. When multiple antennas are available at the relay, the transmission from both sources to the relay in the MA phase can be interpreted as a multiple-input multiple-output (MIMO) system, which allows the application of MIMO detection technologies to separate the two cross-interfered data streams from the sources due to enhanced spatial degrees of freedom. In [6] the optimal maximum likelihood (ML) detector is applied in uncoded MIMO systems, whereas linear detectors applying the zero-forcing (ZF) and minimum mean squared error (MMSE) criteria are considered in [7]. The separately detected messages are further network coded and broadcast in the $\mathrm{BC}$ phase. Additionally, the mutual information of MIMO detection techniques is referred to [8].
In this paper, we concentrate on two-phase two-way relaying systems using XOR-based PLNC and coded OFDM transmission. The analysis from the APP-based decoding schemes in [4] is extended to the multiple-antenna relay scenario. Furthermore, the impact of employing multiple antennas at the relay on the MA phase comparing to the singl-antenna relay scenario is investigated for the different APP-based schemes and several common MIMO detection schemes with respect to mutual information (MI).

The remainder of this paper is organized as follows. The system model is depicted in Section II. The APP-based detectors and several common MIMO detectors with multipleantenna relay are introduced in Section III and Section IV, respectively. Numerical simulation results are presented in Section $\mathrm{V}$, which show comparisons between different schemes with respect to mutual information and error rate performance. Section VI concludes the paper.

\section{SYSTEM DESCRIPTION}

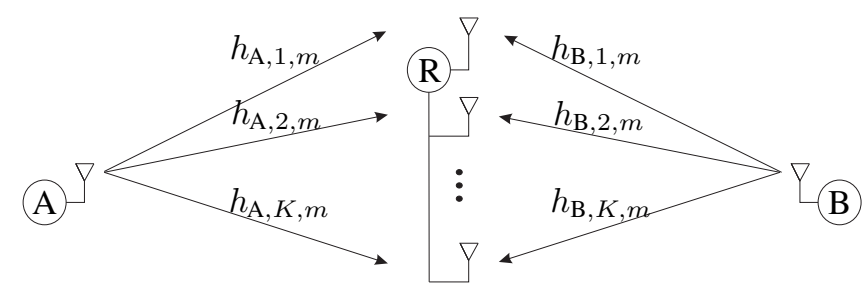

Fig. 1. A two-way relaying network where sources A and B transmit simultaneously to relay $\mathrm{R}$ in the MA phase. Both sources are equipped with one antenna and the relay is equipped with $K$ antennas.

We consider a two-way relaying network shown in Fig. 1, where two sources $\mathrm{A}$ and $\mathrm{B}$ exchange messages with each other helped by a relay $\mathrm{R}$. Both sources are equipped with a single antenna, whereas the relay with $K$ antennas. The two-phase protocol is adopted using XOR-based PLNC and coded orthogonal frequency division multiplexing (OFDM) transmissions. In the MA phase, $\mathrm{A}$ and $\mathrm{B}$ encode their binary information words $b_{A}$ and $b_{B}$ with the same linear code of rate $R_{\mathrm{C}}$ resulting in the codewords $\mathbf{c}_{\mathrm{A}}$ and $\mathbf{c}_{\mathrm{B}}$. Subsequently, the codewords are mapped to OFDM frames that contain symbollevel vectors $\mathrm{s}_{\mathrm{A}}$ and $\mathrm{s}_{\mathrm{B}}$ using a finite alphabet $\mathcal{A}$ with cardinality $M$, which are transmitted to the relay simultaneously. Denoting $N_{\mathrm{C}}$ the total number of subcarriers in one OFDM 
frame, the superimposed received signal $y_{m, k}$ for the $m$ th subcarrier on the $k$ th antenna at $\mathrm{R}$ yields

$$
y_{k, m}=h_{\mathrm{A}, k, m} s_{\mathrm{A}, m}+h_{\mathrm{B}, k, m} s_{\mathrm{B}, m}+n_{k, m}
$$

with $m=1,2, \cdots, N_{\mathrm{C}}$ and $k=1,2, \cdots, K$. Therefore, the MIMO system equation for the $m$ th subcarrier yields

$$
\mathbf{y}_{m}=\mathbf{H}_{m} \mathbf{s}_{m}+\mathbf{n}_{m},
$$

where $\mathbf{s}_{m}=\left[\begin{array}{ll}s_{\mathrm{A}, m} & s_{\mathrm{B}, m}\end{array}\right]^{T}$ and $\mathbf{H}_{m}$ is the $K \times 2$ MIMO channel matrix. The frequency selective channels are Rayleigh block fading containing $N_{\mathrm{H}}$ equal power taps in time domain. Correspondingly, $h_{\mathrm{A}, k, m}$ and $h_{\mathrm{B}, k, m}$ in (1) represent the channel coefficients of the two uplinks in frequency domain with variance $\sigma_{h}^{2}=1 / N_{\mathrm{H}}$. Furthermore, the complex additive white Gaussian noise (AWGN) term $n_{k, m}$ has zero mean and variance $\sigma_{n}^{2}$. Note that the subcarrier index $m$ is omitted in the sequel for the sake of simplicity.

Upon reception relay $\mathrm{R}$ estimates the XOR network coded packet $\mathbf{c}_{\mathrm{R}}$ from the receive signal using different detection and decoding schemes. Subsequently, $\mathbf{c}_{\mathrm{R}}$ is modulated and broadcast to the sources in the $\mathrm{BC}$ phase by, e.g., spacetime block codes (STBC) that exploits spatial diversity with multiple transmit antennas. Finally, each source estimates $\hat{\mathbf{c}}_{\mathrm{R}}$ and applies XOR operation again to remove the selfinterference since the sources know what they transmitted in the MA phase. In this paper, we focus on the critical MA phase as it causes error propagation in the $\mathrm{BC}$ phase.

\section{APP-BASED DETECTORS}

\section{A. APPs in MIMO Systems}

In this section the decoding schemes based on a-posterior probability (APP) detectors introduced in [4] with singleantenna relay are extended to a multiple-antenna scenario. The APP that $\mathbf{s}$ is transmitted conditioned on receiving $\mathbf{y}$ is represented as

$$
\operatorname{Pr}\{\mathbf{s} \mid \mathbf{y}\}=\frac{\operatorname{Pr}\{\mathbf{y} \mid \mathbf{s}\} \operatorname{Pr}\{\mathbf{s}\}}{\operatorname{Pr}\{\mathbf{y}\}}=\frac{\operatorname{Pr}\{\mathbf{y} \mid \mathbf{s}\}}{\sum_{\forall \mathbf{s}} \operatorname{Pr}\{\mathbf{y} \mid \mathbf{s}\}}
$$

by applying the Bayes' rule, where the following equation holds for fixed channel gain and complex Gaussian noise [8]

$$
\operatorname{Pr}\{\mathbf{y} \mid \mathbf{s}\}=\frac{1}{\left(\pi \sigma_{n}^{2}\right)^{K}} \exp \left\{-\frac{1}{\sigma_{n}^{2}}\|\mathbf{y}-\mathbf{H} \mathbf{s}\|^{2}\right\} .
$$

Additionally, since equal a-priori probabilities are assumed at the sources, $\operatorname{Pr}\{\mathbf{s}\}=\frac{1}{M^{2}}$ holds, which indicates that each transmit symbol vector $\mathrm{s}$ appears with probability $\frac{1}{4}$ for BPSK and $\frac{1}{16}$ for QPSK.

\section{B. Separate Decoding (SDC)}

For a MA channel the individual messages from source A and $\mathrm{B}$ can be estimated separately using the APPs defined in (3). Specifically, the log-likelihood ratio $L_{\mathrm{A}}$ for each code bit $c_{\mathrm{A}}$ is calculated as

$$
L_{\mathrm{A}}=\ln \frac{\operatorname{Pr}\left\{c_{\mathrm{A}}=0 \mid \mathbf{y}\right\}}{\operatorname{Pr}\left\{c_{\mathrm{A}}=1 \mid \mathbf{y}\right\}}=\ln \frac{\sum_{\mathbf{s} \in \mathcal{D}_{\mathrm{S}}^{0}} \operatorname{Pr}\{\mathbf{s} \mid \mathbf{y}\}}{\sum_{\mathbf{s} \in \mathcal{D}_{\mathrm{S}}^{1}} \operatorname{Pr}\{\mathbf{s} \mid \mathbf{y}\}} .
$$

Here the sets $\mathcal{D}_{\mathrm{S}}^{0}$ and $\mathcal{D}_{\mathrm{S}}^{1}$ contain all symbol pairs $\mathbf{s}=\left[\begin{array}{ll}s_{\mathrm{A}} & s_{\mathrm{B}}\end{array}\right]^{T}$ with the involved bit $c_{\mathrm{A}}$ equal to 0 and 1 , respectively. Similarly, the LLR $L_{\mathrm{B}}$ for the code bit $c_{\mathrm{B}}$ can be calculated according to (5). Thereafter, the LLRs are sent to the channel decoder to estimate $\hat{\mathbf{c}}_{\mathrm{A}}$ and $\hat{\mathbf{c}}_{\mathrm{B}}$ separately, followed by network coding to produce $\mathbf{c}_{\mathrm{R}}=\hat{\mathbf{c}}_{\mathrm{A}} \oplus \hat{\mathbf{c}}_{\mathrm{B}}$.

The mutual information between the individual bitwise signal, e.g., $c_{\mathrm{A}}$ and the received symbol vector $\mathbf{y}$ is calculated as [5]

$$
\begin{aligned}
C_{\mathrm{S}, \mathrm{A}}= & I\left(c_{\mathrm{A}} ; \mathbf{y}\right) \\
= & \sum_{c_{\mathrm{A}}=i} \int_{-\infty}^{\infty} \operatorname{Pr}\left\{c_{\mathrm{A}}=i, \mathbf{y}\right\} \log _{2} \frac{\operatorname{Pr}\left\{c_{\mathrm{A}}=i, \mathbf{y}\right\}}{\operatorname{Pr}\left\{c_{\mathrm{A}}=i\right\} \operatorname{Pr}\{\mathbf{y}\}} \mathrm{d} \mathbf{y} \\
= & \frac{1}{M^{2}} \sum_{c_{\mathrm{A}}=i} \int_{-\infty}^{\infty} \sum_{\mathbf{s} \in \mathcal{D}_{\mathrm{S}}^{i}} \operatorname{Pr}\{\mathbf{y} \mid \mathbf{s}\} \\
& \cdot \log _{2} \frac{\sum_{\mathbf{s} \in \mathcal{D}_{\mathrm{S}}^{i}} \operatorname{Pr}\{\mathbf{y} \mid \mathbf{s}\}}{\operatorname{Pr}\left\{c_{\mathrm{A}}=i\right\} \sum_{\forall \mathbf{s}} \operatorname{Pr}\{\mathbf{y} \mid \mathbf{s}\}} \mathrm{d} \mathbf{y}
\end{aligned}
$$

with $i=0,1$ and $\operatorname{Pr}\left\{c_{\mathrm{A}}=i\right\}=\frac{1}{2}$. Note that (6) is the subcarrierwise mutual information and relates to deterministic channel coefficients, which can be solved numerically by averaging over sufficient channel realizations. The performance of SDC to estimate the network coded signal for one OFDM frame is upper-bounded by

$$
C_{\mathrm{S}}=\min \left\{\sum_{m} C_{\mathrm{S}, \mathrm{A}, m}, \sum_{m} C_{\mathrm{S}, \mathrm{B}, m}\right\} .
$$

\section{Joint Channel Decoding and Network Coding (JCNC)}

It is noted that the relay is not interested in the individual messages from the sources but only forwards the XORed packet in the BC phase. Therefore, JCNC can be applied which calculates the LLR value $L_{\mathrm{A} \oplus \mathrm{B}}$ for the XORed code bit $c_{\mathrm{A} \oplus \mathrm{B}}=c_{\mathrm{A}} \oplus c_{\mathrm{B}}$ directly using the APPs in (3), which is given by

$$
L_{\mathrm{A} \oplus \mathrm{B}}=\ln \frac{\operatorname{Pr}\left\{c_{\mathrm{A} \oplus \mathrm{B}}=0 \mid \mathbf{y}\right\}}{\operatorname{Pr}\left\{c_{\mathrm{A} \oplus \mathrm{B}}=1 \mid \mathbf{y}\right\}}=\ln \frac{\sum_{\mathbf{s} \in \mathcal{D}_{\mathrm{J}}^{0}} \operatorname{Pr}\{\mathbf{s} \mid \mathbf{y}\}}{\sum_{\mathbf{s} \in \mathcal{D}_{\mathrm{J}}^{1}} \operatorname{Pr}\{\mathbf{s} \mid \mathbf{y}\}} .
$$

Here $\mathcal{D}_{\mathrm{J}}^{0}$ and $\mathcal{D}_{\mathrm{J}}^{1}$ contain all the symbol pairs with $c_{\mathrm{A} \oplus \mathrm{B}}=0$ and $c_{\mathrm{A} \oplus \mathrm{B}}=1$, respectively. Note that for JCNC both sources have to apply the same channel code such that the XORed packet is still a valid codeword in the codebook.

The performance bound for JCNC is determined by the mutual information between the network coded bit $c_{\mathrm{A} \oplus \mathrm{B}}$ and the received symbol vector $\mathbf{y}[5]$

$$
\begin{aligned}
C_{\mathrm{J}}= & I\left(c_{\mathrm{A} \oplus \mathrm{B}} ; \mathbf{y}\right) \\
= & \sum_{c_{\mathrm{A} \oplus \mathrm{B}}=i} \int_{-\infty}^{\infty} \operatorname{Pr}\left\{c_{\mathrm{A} \oplus \mathrm{B}}=i, \mathbf{y}\right\} \log _{2} \frac{\operatorname{Pr}\left\{c_{\mathrm{A} \oplus \mathrm{B}}=i, \mathbf{y}\right\}}{\operatorname{Pr}\left\{c_{\mathrm{A} \oplus \mathrm{B}}=i\right\} \operatorname{Pr}\{\mathbf{y}\}} \mathrm{d} \mathbf{y} \\
= & \frac{1}{M^{2}} \sum_{c_{\mathrm{A} \oplus \mathrm{B}}=i} \int_{-\infty}^{\infty} \sum_{\mathbf{s} \in \mathcal{D}_{\mathrm{J}}^{i}} \operatorname{Pr}\{\mathbf{y} \mid \mathbf{s}\} \\
& \cdot \log _{2} \frac{\sum_{\mathbf{s} \in \mathcal{D}_{\mathrm{J}}^{i}} \operatorname{Pr}\{\mathbf{y} \mid \mathbf{s}\}}{\operatorname{Pr}\left\{c_{\mathrm{A} \oplus \mathrm{B}}=i\right\} \sum_{\forall \mathbf{s}} \operatorname{Pr}\{\mathbf{y} \mid \mathbf{s}\}} \mathrm{dy}
\end{aligned}
$$


with $\operatorname{Pr}\left\{c_{\mathrm{A} \oplus \mathrm{B}}=i\right\}=\frac{1}{2}$. Note that (9) represents the subcarrierwise mutual information, which needs to be averaged over $m$ to achieve the mutual information for one OFDM frame.

\section{Generalized JCNC (G-JCNC)}

It has been shown in [3] that the useful information provided by the two channel codes applied at the sources is not fully exploited by JCNC, which motivates a generalized sumproduct algorithm for LDPC codes, as proposed in [4]. In the G-JCNC scheme, the APPs defined in (3) are directly used and updated iteratively in the non-binary belief propagation decoder instead of the LLRs.

The performance for G-JCNC is theoretically evaluated by the mutual information between the transmit signal vector $\mathbf{s}$ and the received signal vector $\mathbf{y}[5]$

$$
\begin{aligned}
C_{\mathrm{G}}^{\prime} & =I(\mathbf{s} ; \mathbf{y}) \\
& =\sum_{\mathbf{s}} \int_{-\infty}^{\infty} \operatorname{Pr}\{\mathbf{s}, \mathbf{y}\} \log _{2} \frac{\operatorname{Pr}\{\mathbf{s}, \mathbf{y}\}}{\operatorname{Pr}\{\mathbf{s}\} \operatorname{Pr}\{\mathbf{y}\}} \mathrm{d} \mathbf{y} \\
& =\frac{1}{M^{2}} \sum_{\mathbf{s}} \int_{-\infty}^{\infty} \operatorname{Pr}\{\mathbf{y} \mid \mathbf{s}\} \log _{2} \frac{M^{2} \operatorname{Pr}\{\mathbf{y} \mid \mathbf{s}\}}{\sum_{\forall \mathbf{s}} \operatorname{Pr}\{\mathbf{y} \mid \mathbf{s}\}} \mathrm{d} \mathbf{y} .
\end{aligned}
$$

Since $C_{\mathrm{G}}^{\prime}$ corresponds to the sum-rate of the MA channel, a fair comparison with $C_{\mathrm{S}}$ and $C_{\mathrm{J}}$ is achieved by defining $C_{\mathrm{G}}=C_{\mathrm{G}}^{\prime} / 2$. Similarly, $C_{\mathrm{G}}$ has to be averaged over $m$ in OFDM systems.

\section{COMmON MIMO Detectors}

\section{A. Mutual Information}

The APP-based detectors introduced in the previous section work for arbitrary number of antennas at the relay but the complexity grows exponentially with higher modulation alphabets. In our system setup, the MIMO channel in the MA phase allows the application of common MIMO detection schemes with lower computational effort when $K \geq 2$. In this section, several MIMO detection schemes are investigated that estimate s, followed by network coding to generate the XORed packet for broadcasting.

Denoting the filtered signal vector as $\tilde{\mathbf{s}}=\left[\begin{array}{ll}\tilde{s}_{\mathrm{A}} & \tilde{s}_{\mathrm{B}}\end{array}\right]^{T}=\mathbf{G y}$, where $\mathbf{G}$ represents the filter matrix for MIMO detection, the mutual information for a $2 \times K$ MIMO system to estimate the nework coded signal using MIMO detection techniques in OFDM systems is given as

$$
C_{\mathrm{MIMO}}=\min \left\{\sum_{m} I\left(s_{\mathrm{A}, m} ; \tilde{s}_{\mathrm{A}, m}\right), \sum_{m} I\left(s_{\mathrm{B}, m} ; \tilde{s}_{\mathrm{B}, m}\right)\right\} .
$$

Furthermore, let the equivalent channel coefficients and noise variance for the filtered signal vector be denoted as $\mathbf{h}_{\mathrm{eq}}=$ $\left[h_{\mathrm{eq}, \mathrm{A}} h_{\mathrm{eq}, \mathrm{B}}\right]^{T}$ and $\boldsymbol{\sigma}_{n, \mathrm{eq}}^{2}=\left[\sigma_{n, \mathrm{eq}, \mathrm{A}}^{2} \sigma_{n, \mathrm{eq}, \mathrm{B}}^{2}\right]^{T}$, respectively. The subcarrierwise per-layer mutual information, e.g. $I\left(s_{\mathrm{A}} ; \tilde{s}_{\mathrm{A}}\right)$, is computed numerically in (12) according to [9], which calculated mutual information for transmitting finite alphabets over AWGN channels with deterministic channel gain. Note that the remaining interference in $\tilde{\mathbf{s}}$ is treated as Gaussian noise, as discussed in more details in [8]. In the following subsections, different MIMO detection schemes are introduced with $\mathbf{h}_{\mathrm{eq}}$ and $\boldsymbol{\sigma}_{n, \mathrm{eq}}^{2}$ defined to calculate the corresponding mutual information.

\section{B. Linear Equalization (LE)}

The filter matrix $\mathbf{G}$ for linear detectors is given as

$$
\mathbf{G}= \begin{cases}\mathbf{H}^{+}=\left(\mathbf{H}^{H} \mathbf{H}\right)^{-1} \mathbf{H} & \mathrm{ZF} \\ \underline{\mathbf{H}}^{+}=\left(\underline{\mathbf{H}}^{H} \underline{\mathbf{H}}\right)^{-1} \underline{\mathbf{H}} & \mathrm{MMSE}\end{cases}
$$

for the zero-forcing (ZF) and minimum mean squared error (MMSE) criteria, respectively. The operator $(\cdot)^{+}$denotes the pseudo inverse of a matrix and $\underline{\mathbf{H}}$ represents the extended channel matrix given as

$$
\underline{\mathbf{H}}=\left[\begin{array}{c}
\mathbf{H} \\
\sigma_{n} \mathbf{I}_{2}
\end{array}\right] \text {. }
$$

The estimation errors of different layers are determined by the main diagonal terms of the error covariance matrix, which has the following form

$$
\boldsymbol{\Phi}= \begin{cases}\sigma_{n}^{2}\left(\mathbf{H}^{H} \mathbf{H}\right)^{-1} & \mathrm{ZF} \\ \sigma_{n}^{2}\left(\underline{\mathbf{H}}^{H} \underline{\mathbf{H}}\right)^{-1} & \text { MMSE } .\end{cases}
$$

Denoting $\mathbf{g}_{j}$ the $j$ th row of $\mathbf{G}, j=1,2$, the equivalent channel coefficients and noise variance are given by

$$
\begin{aligned}
& \mathbf{h}_{\mathrm{eq}}= \begin{cases}\mathbf{1}_{2 \times 1} & \mathrm{ZF} \\
\operatorname{dg}\{\mathbf{G H}\} & \mathrm{MMSE}\end{cases} \\
& \boldsymbol{\sigma}_{n, \mathrm{eq}}^{2}= \begin{cases}{\left[\begin{array}{c}
\left\|\mathbf{g}_{1}\right\|^{2} \\
\left\|\mathbf{g}_{2}\right\|^{2}
\end{array}\right] \sigma_{n}^{2}} & \mathrm{ZF} \\
{\left[\begin{array}{c}
\left\|\mathbf{g}_{1}\right\|^{2} \\
\left\|\mathbf{g}_{2}\right\|^{2}
\end{array}\right] \sigma_{n}^{2}+\left[\begin{array}{c}
\left|\epsilon_{1}\right|^{2} \\
\left|\epsilon_{2}\right|^{2}
\end{array}\right]} & \text { MMSE , }\end{cases}
\end{aligned}
$$

where the operator $\mathrm{dg}(\cdot)$ collects the diagonal terms of a matrix to form a column vector. $\epsilon_{1}$ and $\epsilon_{2}$ represent the non-diagonal terms of $\mathbf{G H}$ for the 1st layer and the 2nd layer, respectively. The mutual information of linear detectors can be calculated by incorporating (16) into (12).

\section{Successive Interference Cancellation (SIC) based on $Q R$ Decomposition (QRD)}

The uplink $2 \times K$ MIMO channel $\mathbf{H}$ can be decomposed into $\mathbf{H}=\mathbf{Q R}$, where $\mathbf{Q}$ denotes a $K \times 2$ matrix with orthonormal columns and $\mathbf{R}$ denotes a $2 \times 2$ upper triangular matrix under the $\mathrm{ZF}$ criterion. In case of MMSE, QRD is applied to the extended channel matrix (14) as $\underline{\mathbf{H}}=\underline{\mathbf{Q}} \underline{\mathbf{R}}$. Employing $\mathbf{Q}^{H}$ and $\underline{\mathbf{Q}}^{H}$ as the filter matrix for ZF and MMSE, respectively, yields the filter output [10]

$$
\tilde{\mathbf{s}}= \begin{cases}\mathbf{R} \mathbf{s}+\mathbf{Q}^{H} \mathbf{n} & \mathrm{ZF} \\ \underline{\mathbf{R}} \mathbf{s}-\sigma_{n} \mathbf{Q}_{2}^{H} \mathbf{s}+\mathbf{Q}_{1}^{H} \mathbf{n} & \mathrm{MMSE},\end{cases}
$$

where $\underline{\mathbf{Q}}$ is partitioned into $K \times 2$ matrix $\mathbf{Q}_{1}$ and $2 \times 2$ lower triangular matrix $\mathbf{Q}_{2}$. Note that the statistical property of the equivalent noise is changed for MMSE since the columns of 


$$
I\left(s_{\mathrm{A}} ; \tilde{s}_{\mathrm{A}}\right)=\log _{2} M-\mathbb{E}_{\mathbf{H}, \mathbf{n}}\left[\frac{1}{M} \sum_{s_{\mathrm{A}}} \log _{2}\left(1+\sum_{s_{\mathrm{A}}^{\prime} \neq s_{\mathrm{A}}} \frac{\exp \left\{-\frac{1}{\sigma_{n, \mathrm{eq}, \mathrm{A}}}\left|\tilde{s}_{\mathrm{A}}^{\prime}-h_{\mathrm{eq}, \mathrm{A}} s_{\mathrm{A}}^{\prime}\right|^{2}\right\}}{\exp \left\{\frac{1}{\sigma_{n, \mathrm{eq}, \mathrm{A}}^{2}}\left|\tilde{s}_{\mathrm{A}}-h_{\mathrm{eq}, \mathrm{A}} s_{\mathrm{A}}\right|^{2}\right\}}\right)\right]
$$

$\mathbf{Q}_{1}$ are not orthonormal. Due to the upper triangular structure of $\mathbf{R}$ and $\underline{\mathbf{R}}$, the 2 nd layer is detected first, whose impact is subtracted when detecting the 1st layer subsequently. Therefore, the equivalent channel coefficient and noise variance vectors are given by

$$
\begin{aligned}
\mathbf{h}_{\mathrm{eq}} & = \begin{cases}\operatorname{dg}\{\mathbf{R}\} & \mathrm{ZF} \\
\operatorname{dg}\{\underline{\mathbf{R}}\}-\operatorname{dg}\left\{\sigma_{n} \mathbf{Q}_{2}^{H}\right\} & \text { MMSE }\end{cases} \\
\boldsymbol{\sigma}_{n, \mathrm{eq}}^{2} & =\left\{\begin{array}{c}
\mathbf{1}_{2 \times 1} \sigma_{n}^{2} \\
{\left[\begin{array}{c}
\left\|\mathbf{q}_{1}\right\|^{2} \\
\left\|\mathbf{q}_{2}\right\|^{2}+\left|\epsilon_{3}\right|^{2}
\end{array}\right] \sigma_{n}^{2}}
\end{array}\right.
\end{aligned}
$$

Here $\mathbf{q}_{j}$ represents the $j$ th row of $\mathbf{Q}_{1}^{H} \cdot \epsilon_{3}$ denotes the lower triangular term of $\mathbf{Q}_{2}^{H}$. The mutual information for $\mathrm{QRD}$ based SIC can be calculated by using (18) in (12).

The bottleneck of SIC is that the erroneous decisions of the detected layer will be propagated to the layer to be detected. To this end, the layer with the higher reliability should be detected first to suppress error propagation. Note that the same detection order is required on all subcarriers due to the fact that channel coding is applied to the OFDM frames at source A and B individually. One possible approach as indicated in [11] is, that the layer with the smaller average estimation error $\bar{\Phi}_{j}$ over one OFDM frame is selected as the target layer, which is defined as

$$
\bar{\Phi}_{j}=\frac{1}{N_{\mathrm{C}}} \sum_{m=1}^{N_{\mathrm{C}}}\left[\boldsymbol{\Phi}_{m}\right]_{j, j} .
$$

Here $\boldsymbol{\Phi}_{m}$ denotes the error covariance matrix on the $m$ th subcarrier defined in (15). The operator $[\cdot]_{j, j}$ takes the main diagonal term of a matrix for the $j$ the layer with $j=1,2$.

\section{Numerical Results}

A two-phase two-way relaying system is considered where the relay is located in the middle of the two sources and the three nodes are on a line. We concentrate on the critical MA phase over multiple-path Rayleigh block fading channels using OFDM with $N_{\mathrm{H}}=5$ and $N_{\mathrm{C}}=1024$ subcarriers. In the link level simulations, both sources use QPSK modulation and optimized irregular LDPC codes with codeword length $n=$ 16200. 100 iterations are employed for both binary and nonbinary decoding.

The mutual information for the APP based schemes with different number of antennas at the relay are shown in Fig. 2. It can be observed that SDC outperforms JCNC whereas GJCNC achieves the best performance over the whole code rate region. JCNC approaches SDC with higher code rates. Obviously, more receive antennas at the relay lead to higher mutual information. Of specific interest is that with growing $K$ SDC approaches G-JCNC, e.g., the loss of mutual information for SDC to G-JCNC is reduced from $4 \mathrm{~dB}$ for $K=1$ to $1 \mathrm{~dB}$ for $K=5$ at the mutual information equal to 1 . This implies that SDC may be beneficial with multiple-antenna relay in implementation aspects due to lower complexity.

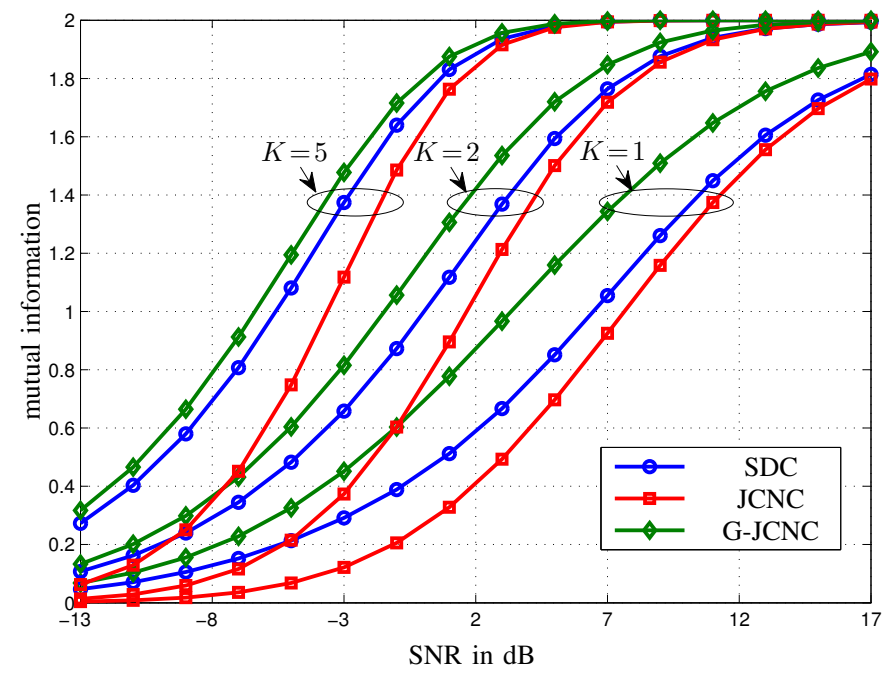

Fig. 2. Mutual information for the SDC, JCNC and G-JCNC schemes over multi-path fading channels using OFDM. The relay is equipped with different number of antennas, i.e., $K=1,2,5$.

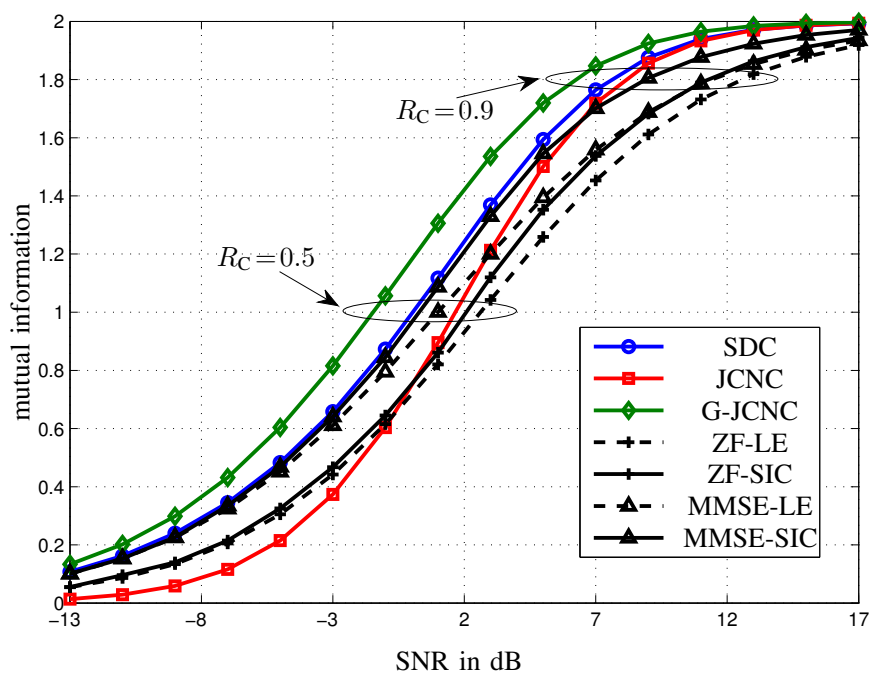

Fig. 3. Mutual information for the APP based schemes and different MIMO detection schemes over multi-path fading channels using OFDM. The relay is equipped with $K=2$ antennas.

In Fig. 3 the APP based schemes are compared with different MIMO detection schemes with respect to mutual information for $K=2$ antennas at the relay. Note that the 
QRD based SIC MIMO detectors use the sorting criterion (19). It is shown that SIC achieves improved mutual information especially with high code rates compared to the linear detectors, which corresponds to the observations in [8]. Furthermore, the MMSE based SIC performs close to SDC with low and dedium code rates, but the computational efforts are lower than the APP based schemes.

The frame error rate (FER) performance of the APP based schemes and the MMSE-SIC scheme is shown in Fig. 4 and Fig. 5 for code rate $R_{\mathrm{C}}=0.5$ and $R_{\mathrm{C}}=0.9$, respectively. Note that the FER refers to the XORed packets. Compared to Fig. 2 and Fig. 3, it leads to the same conclusion that introducing multiple antenna decreases the performance gap between SDC and G-JCNC. Furthermore, SDC is only slightly better than MMSE-SIC with $R_{\mathrm{C}}=0.5$ but still outperforms MMSE-SIC greatly with high code rate, e.g., $R_{\mathrm{C}}=0.9$. It is also verified that JCNC performs close to SDC with high code rates.

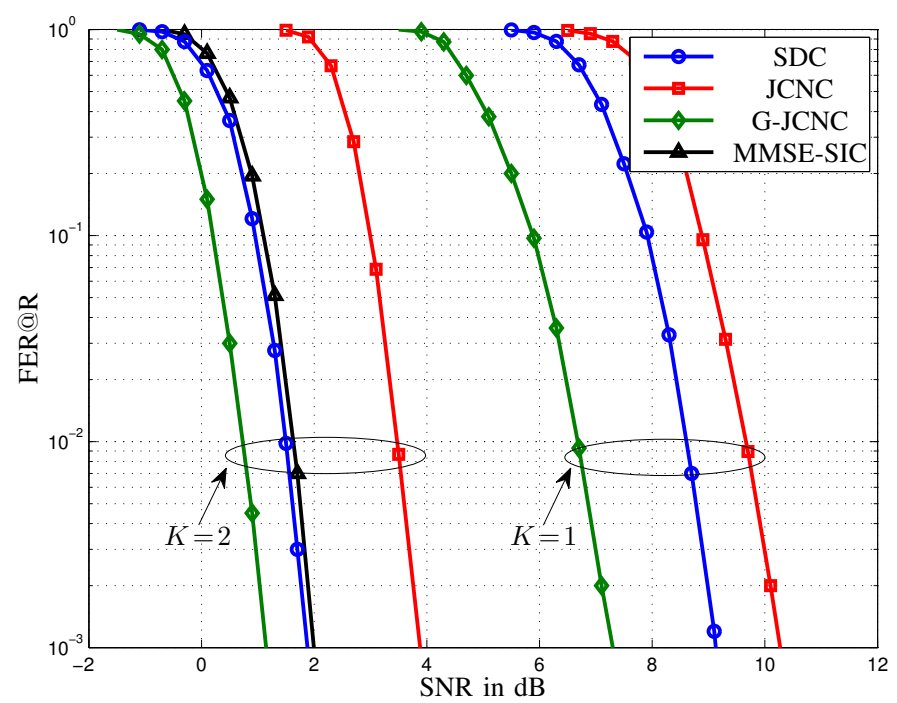

Fig. 4. FER performance at the relay for the APP based schemes and the MMSE-SIC scheme over multi-path fading channels using OFDM. The LDPC codeword length is set to $n=16200,100$ iterations, $R_{\mathrm{C}}=0.5$, QPSK

\section{CONCLUDING REMARKS}

Two-phase two-way relaying using XORed physical layer network coding in coded OFDM systems was investigated in this paper. The employment of multiple antennas at the relay allows the application of MIMO detection techniques in the multiple-access (MA) phase. We addressed the impact of multiple antennas for signal detection on different detection and decoding schemes, which was studied with respect to finite alphabet constrained mutual information. The analytical researches were verified by link level simulations.

\section{ACKNOWLEDGEMENT}

This work was supported in part by the German Research Foundation (DFG) under grant Wu 499/8-1 within the priority program "Communication in Interference Limited Networks (COIN)", SPP 1397.

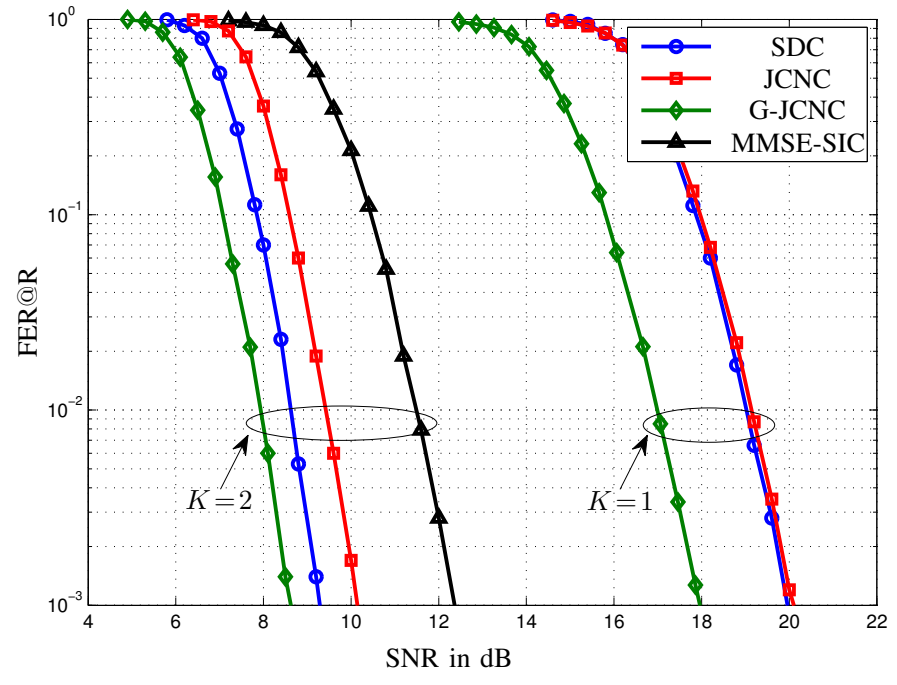

Fig. 5. FER performance at the relay for the APP based schemes and the MMSE-SIC scheme over multi-path fading channels using OFDM. The LDPC codeword length is set to $n=16200,100$ iterations, $R_{\mathrm{C}}=0.9$, QPSK.

\section{REFERENCES}

[1] S. Zhang, S .C. Liew, and P. Lam, "Hot Topic: Physical Layer Network Coding," in International Conference on Mobile Computing and Networking (MobiCom), Log Angeles, CA, USA, Mar. 2006.

[2] T. Koike-Akino, P. Popovski, and V. Tarokh, "Optimized Constellations for Two-Way Wireless Relaying with Physical Network Coding," IEEE Journal on Selected Areas in Communications, vol. 27, no. 5, pp. 773787, Jun. 2009.

[3] S. Zhang and S. C. Liew, "Channel Coding and Decoding in a Relay System Operated with Physical-Layer Network Coding," IEEE Journal on Selected Areas in Communications, vol. 27, no. 5, pp. 788-796, Oct. 2009.

[4] D. Wübben, "Joint Channel Decoding and Physical-Layer Network Coding in Two-Way QPSK Relay Systems by a Generalized Sum-Product Algorithm," in 7th International Symposium on Wireless Communication Systems (ISWCS'10), York, United Kingdom, Sept. 2010.

[5] S. Pfletschinger, "A Practical Physical-Layer Network Coding Scheme for the Uplink of the Two-Way Relay Channel," in 45th Asilomar Conference on Signals, Systems and Computers (ASILOMAR'11), Monterey, CA, USA, Nov. 2011.

[6] Z. Zhou and B. Vucetic, "An Optimized Network Coding Scheme in Two-Way Relay Channels with Multiple Relay Antennas," in IEEE 20th International Symposium on Personal, Indoor and Mobile Radio Communications (PIMRC'09), Tokyo, Japan, Sept. 2009.

[7] D. Xu, Z. Bai, A. Waadt, G. H. Bruck, and P. Jung, "Combining MIMO with Network Coding: A Viable Means to Provide Multiplexing and Diversity in Wireless Relay Networks," in IEEE International Conference on Communications (ICC'10), Cape Town, South Africa, May 2010.

[8] E. Ohlmer, U. Wachsmann, and G. Fettweis, "Mutual Information of MIMO Transmission over Correlated Channels with Finite Symbol Alphabet and Link Adaptation," in IEEE Global Communications Conference (GLOBECOM'10), Miami, FL, USA, Dec. 2010.

[9] G. Ungerboeck, "Channel Coding with Multilevel/Phase Signals," IEEE Transactions on Information Theory, vol. 28, no. 1, pp. 55-67, Jan. 1982.

[10] D. Wübben, R. Böhnke, V. Kühn, and K. D. Kammeyer, "MMSE Extension of V-BLAST based on Sorted QR Decomposition," in IEEE 58th Vehicular Technology Conference (VTC'03-Fall), Orlando, FL, USA, Oct. 2003.

[11] D. Wübben and K. D. Kammeyer, "Low Complexity Successive Interference Cancellation for Per-Antenna-Coded MIMO-OFDM Schemes by Applying Parallel-SQRD," in IEEE 63rd Vehicular Technology Conference (VTC'06-Spring), Melbourne, Australia, May 2006. 